\title{
Proteinase 3-antineutrophil cytoplasmic antibody-positive necrotizing crescentic glomerulonephritis complicated by infectious endocarditis: a case report
}

Katsunori Yanai ${ }^{1+}$, Yoshio Kaku ${ }^{1 \dagger}$, Keiji Hirai ${ }^{1 *}$, Shohei Kaneko ${ }^{1}$, Saori Minato ${ }^{1}$, Yuko Mutsuyoshi ${ }^{1}$, Hiroki Ishii ${ }^{1}$, Taisuke Kitano', Mitsutoshi Shindo', Haruhisa Miyazawa', Kiyonori Ito', Yuichiro Ueda', Masahiro Hiruta?', Susumu Ookawara ${ }^{1}$, Yoshihiko Ueda ${ }^{3}$ and Yoshiyuki Morishita ${ }^{*}$

\begin{abstract}
Background: Proteinase 3-antineutrophil cytoplasmic antibody has been reported to be positive in 5-10\% of cases of renal injury complicated by infective endocarditis; however, histological findings have rarely been reported for these cases.

Case presentation: A 71-year-old Japanese man with a history of aortic valve replacement developed rapidly progressive renal dysfunction with gross hematuria and proteinuria. Blood analysis showed a high proteinase 3antineutrophil cytoplasmic antibody $(163 \mathrm{lU} / \mathrm{ml})$ titer. Streptococcus species was detected from two separate blood culture bottles. Transesophageal echocardiography detected mitral valve vegetation. Histological evaluation of renal biopsy specimens showed necrosis and cellular crescents in glomeruli without immune complex deposition. The patient met the modified Duke criteria for definitive infective endocarditis. On the basis of these findings, the patient was diagnosed with proteinase 3-antineutrophil cytoplasmic antibody-positive necrotizing crescentic glomerulonephritis complicated by Streptococcus infective endocarditis. His renal disease improved, and his proteinase 3-antineutrophil cytoplasmic antibody titer normalized with antibiotic monotherapy.

Conclusion: Few case reports have described histological findings of proteinase 3-antineutrophil cytoplasmic antibody-positive renal injury complicated with infective endocarditis. We believe that an accumulation of histological findings and treatments is mandatory for establishment of optimal management for proteinase 3antineutrophil cytoplasmic antibody-positive renal injury complicated with infective endocarditis.
\end{abstract}

Keywords: Necrotizing crescentic glomerulonephritis, Infective endocarditis, Proteinase 3-antineutrophil cytoplasmic antibody

\section{Background}

Proteinase 3-antineutrophil cytoplasmic antibody (PR3ANCA) has been reported to be positive in $5-10 \%$ of cases of renal injury complicated by infective endocarditis [1]; however, histological findings have rarely been

\footnotetext{
* Correspondence: keijihirai@kfy.biglobe.ne.jp; ymori@jichi.ac.jp ${ }^{\dagger}$ Katsunori Yanai and Yoshio Kaku are equal first authors.

'Division of Nephrology, First Department of Integrated Medicine, Saitama Medical Center, Jichi Medical University, 1-847 Amanuma-cho, Omiya-ku,

Saitama, Saitama 330-8503, Japan

Full list of author information is available at the end of the article
}

reported for these cases. In addition, the clinical course and optimal treatment have not been fully clarified.

We report a case of a patient with rapidly progressive PR3-ANCA-positive necrotizing crescentic glomerulonephritis complicated by Streptococcus infective endocarditis. The patient's renal disease improved with antibiotic therapy without any immunosuppressive agents, and his PR3-ANCA titer normalized in accordance with improving infective endocarditis.

(c) The Author(s). 2019 Open Access This article is distributed under the terms of the Creative Commons Attribution 4.0 International License (http://creativecommons.org/licenses/by/4.0/), which permits unrestricted use, distribution, and 
Table 1 Laboratory findings upon admission

\begin{tabular}{|c|c|}
\hline $\begin{array}{l}\text { Complete blood count and blood } \\
\text { chemistry }\end{array}$ & Value \\
\hline WBC & $13,600 / \mu \mathrm{L}$ \\
\hline Bands & $2 \%$ \\
\hline Segments & $82 \%$ \\
\hline Eosinophils & $0 \%$ \\
\hline Basophils & $0 \%$ \\
\hline Lymphocytes & $7 \%$ \\
\hline Monocytes & $8 \%$ \\
\hline RBC & $323 \times 10^{4} / \mu \mathrm{L}$ \\
\hline Hemoglobin & $7.6 \mathrm{~g} / \mathrm{dL}$ \\
\hline Hematocrit & $29.2 \%$ \\
\hline Platelet & $12.0 \times 10^{4} / \mu \mathrm{L}$ \\
\hline Total protein & $7.0 \mathrm{~g} / \mathrm{dL}$ \\
\hline Albumin & $2.6 \mathrm{~g} / \mathrm{dL}$ \\
\hline AST & $35 \mathrm{IU} / \mathrm{L}$ \\
\hline ALT & $23 \mathrm{IU} / \mathrm{L}$ \\
\hline CRP & $7.57 \mathrm{mg} / \mathrm{dL}$ \\
\hline $\mathrm{Na}^{+}$ & $130 \mathrm{mmol} / \mathrm{L}$ \\
\hline $\mathrm{K}^{+}$ & $5.3 \mathrm{mmol} / \mathrm{L}$ \\
\hline $\mathrm{Cl}^{-}$ & $101 \mathrm{mmol} / \mathrm{L}$ \\
\hline $\mathrm{Ca}^{2+}$ & $7.7 \mathrm{mg} / \mathrm{dL}$ \\
\hline Phosphate & $3.8 \mathrm{mg} / \mathrm{dL}$ \\
\hline BUN & $25 \mathrm{mg} / \mathrm{dL}$ \\
\hline $\mathrm{Cr}$ & $2.52 \mathrm{mg} / \mathrm{dL}$ \\
\hline eGFR & $20.8 \mathrm{ml} /$ minute $/ 1.73 \mathrm{~m}^{2}$ \\
\hline Uric acid & $6.3 \mathrm{mg} / \mathrm{dL}$ \\
\hline $\mathrm{HbA1c}$ & $6.3 \%$ \\
\hline Glucose & $106 \mathrm{mg} / \mathrm{dL}$ \\
\hline ASO & $73 \mathrm{IU} / \mathrm{mL}$ \\
\hline Hepatitis B antigen & $<0.04$ \\
\hline Hepatitis C antibody & $<0.29$ \\
\hline $\lg G$ & $2692 \mathrm{mg} / \mathrm{dL}$ \\
\hline $\lg A$ & $340 \mathrm{mg} / \mathrm{dL}$ \\
\hline $\lg M$ & $350 \mathrm{mg} / \mathrm{dL}$ \\
\hline Anti-DNA antibody & $<10$ \\
\hline Anti-RNP antibody & Negative \\
\hline Anti-Sm antibody & Negative \\
\hline C3 & $50 \mathrm{mg} / \mathrm{dL}$ \\
\hline C4 & 17 mg/dL \\
\hline $\mathrm{CH} 50$ & $23.4 \mathrm{U} / \mathrm{mL}$ \\
\hline Antinuclear antibody & 160 \\
\hline PR3-ANCA & $163 \mathrm{IU} / \mathrm{mL}$ \\
\hline MPO-ANCA & $<1.0 \mathrm{IU} / \mathrm{mL}$ \\
\hline Anti-GBM antibody & $<2.0 \mathrm{IU} / \mathrm{mL}$ \\
\hline
\end{tabular}

Table 1 Laboratory findings upon admission (Continued)

\begin{tabular}{ll}
$\begin{array}{l}\text { Complete blood count and blood } \\
\text { chemistry }\end{array}$ & Value \\
\hline ESR & $86 \mathrm{~mm} / \mathrm{hour}$ \\
Rheumatoid factor & $86 \mathrm{IU} / \mathrm{mL}$ \\
Urinary analysis & \\
RBC & Numerous (dysmorphic)/HPF \\
WBC & $1-4 / \mathrm{HPF}$ \\
Protein & $0.74 \mathrm{~g} / \mathrm{g} \mathrm{Cr}$ \\
$\beta_{2}$-MG & $12,133 \mathrm{\mu g} / \mathrm{L}$ \\
\hline
\end{tabular}

Abbreviations: $A L T$ alanine aminotransferase, $A S O$ antistreptolysin $\mathrm{O}, A S T$ aspartate aminotransferase, $\beta_{2}-M G \beta_{2}$-microglobulin, $B U N$ blood urea nitrogen, CH50 50\% homolytic unit of complement, $\mathrm{Cr}$ creatinine, CRP C-reactive protein, C3 complement component 3, C4 complement component 4, eGFR estimated glomerular filtration rate, ESR erythrocyte sedimentation rate, GBM antiglomerular basement membrane antibody, HbA1c hemoglobin A1c, HPF high-power field, Ig immunoglobulin, MPO-ANCA myeloperoxidase antineutrophil cytoplasmic antibody, PR3-ANCA proteinase 3 antineutrophil cytoplasmic antibody, RBC red blood cells, RNP ribonucleoprotein, Sm Smith, WBC white blood cells

\section{Case presentation}

Our patient was a 71-year-old Japanese man who had undergone the Bentall procedure and biological aortic valve replacement for the treatment of descending aortic aneurysm and aortic regurgitation at 70 years of age. Thereafter, his renal function had been normal (serum creatinine level, $0.93 \mathrm{mg} / \mathrm{dl}$ ) without hematuria and proteinuria. Two months before admission, he had appetite loss, malaise, and gross hematuria. One month before admission, he noticed purpura on his lower extremities. A laboratory examination conducted by his primary care physician showed anemia (hemoglobin, $9.2 \mathrm{~g} / \mathrm{dl}$ ), thrombocytopenia (platelet count, $\left.10 \times 10^{4} / \mu \mathrm{l}\right)$, hematuria, and proteinuria. Therefore, he was referred to our hospital for further management.

Upon admission, his body temperature was $36.9^{\circ} \mathrm{C}$, and his blood pressure was $120 / 60 \mathrm{mmHg}$. Anemia, edema, and symmetrically distributed palpable purpura of the lower extremities were observed. He had no characteristic physical findings of infective endocarditis, such as Osler nodes, Roth spots, and Janeway lesions. Cardiac auscultation revealed $2 / 6$ systolic reflux murmur at the cardiac apex. Blood analysis showed that the patient's serum creatinine level was elevated at $2.34 \mathrm{mg} / \mathrm{dl}$, and his serum hemoglobin level was reduced at $7.6 \mathrm{~g} / \mathrm{dl}$. Urinalysis showed proteinuria at $0.74 \mathrm{~g} / \mathrm{g} \mathrm{Cr}$ and microscopic hematuria. PR3-ANCA level was elevated at 163 $\mathrm{IU} / \mathrm{ml}$ (normal range, $<10 \mathrm{IU} / \mathrm{ml}$ ). The patient had negative test results for hepatitis $B$ antigen, hepatitis $C$ antibody, cryoglobulin, antistreptolysin $\mathrm{O}$, antinuclear antibody, immune complex, and myeloperoxidaseANCA. Serum complement C3 was mildly decreased, whereas $\mathrm{C} 4$ was normal. Laboratory data obtained at admission are summarized in Table 1. No abnormalities were found in the patient's chest x-ray or electrocardiogram. Streptococcus species was detected from two 
separate blood culture bottles. On the third hospital day, renal biopsy was performed. Histological analysis revealed that $54 \%$ ( 6 of 11) of glomeruli showed partial fibrinoid necrosis with fragmentation of glomerular tufts (Fig. 1a), and 27\% (3 of 11) of glomeruli showed cellular crescents (Fig. 1b). No fibrocellular or fibrous crescents and no endocapillary proliferation were found. The mesangium showed no increase in cells or matrix. The tubulointerstitium partially showed neutrophilic and lymphocytic infiltration in the peritubular capillary and atrophy (Fig. 1c). Fibrinoid necrosis was not observed in vessel walls. Immunofluorescence microscopy showed no deposition of immunoglobulins and complement factors. Electron microscopy showed small amounts of nonspecific electron-dense deposits in subendothelial areas and the paramesangial area. At this point, the patient met the modified Duke criteria for definitive infective endocarditis [2] (mitral valve vegetation on echocardiography, two positive blood cultures of Streptococcus species drawn 3 days apart, glomerulonephritis). On the eighth hospital day, transesophageal echocardiography revealed mitral valve vegetation. On the 12th hospital day, spinal magnetic resonance imaging showed pyogenic spondylitis at T7/T8 and L4/L5. On the basis of these findings, the patient was diagnosed with rapidly progressive PR3-ANCA-positive necrotizing crescentic glomerulonephritis complicated by Streptococcus infective endocarditis. Antibiotic therapy including cefazolin and penicillin $G$ followed by oral administration of ampicillin was provided without immunosuppressive agents. Thereafter, his renal disease, endocarditis, and pyogenic spondylitis improved. He was discharged from our center on the 73rd hospital day. He has since received regular outpatient treatment in our department. At 7 months after discharge, his serum creatinine level had decreased to $1.43 \mathrm{mg} / \mathrm{dl}$, his proteinuria had decreased to $0.15 \mathrm{~g} / \mathrm{g} \mathrm{Cr}$, and his hematuria had decreased to 1.1 red blood cells per high-power field. His PR3ANCA level had decreased to within the normal range (Fig. 2).

\section{Discussion and conclusions}

We report a case of rapidly progressive PR3-ANCApositive necrotizing crescentic glomerulonephritis complicated by Streptococcus infective endocarditis. The patient's renal disease improved with antibiotic monotherapy, which led to normalization of PR3ANCA titer in accordance with improving infective endocarditis.

Renal disease associated with infective endocarditis shows various pathological changes including crescent formation, fibrinoid necrosis, mesangial cell proliferation,

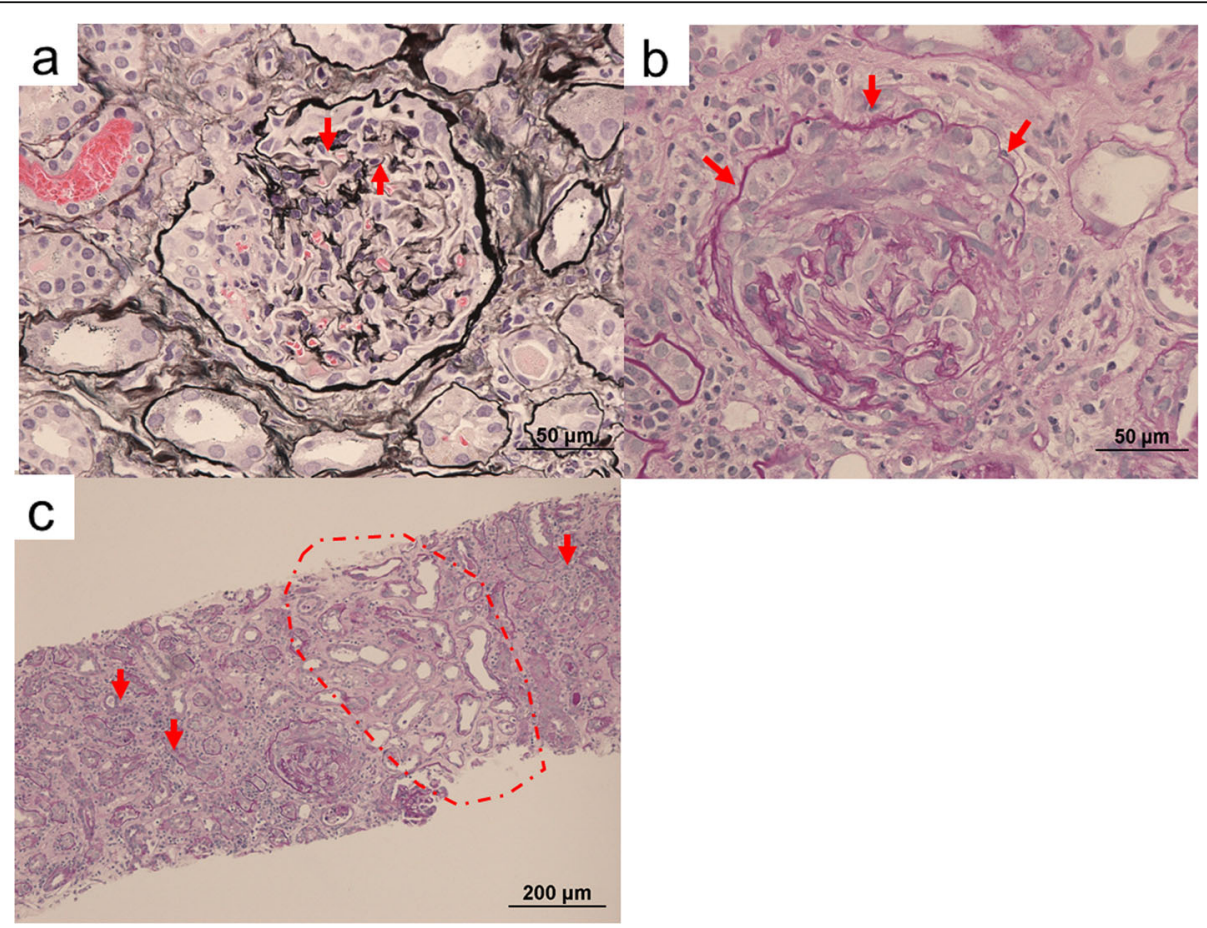

Fig. 1 Renal biopsy findings. a Glomerulus with partial fibrinoid necrosis with fragmentation of glomerular tufts (arrows) (periodic acidmethenamine silver stain; original magnification, 400x). b Glomerulus with cellular crescentic formation (arrows) (periodic acid-Schiff stain; magnification, original magnification, 400x). c Tubulointerstitium with sporadic neutrophil infiltration in the peritubular capillary (arrows) and atrophy (broken line) (periodic acid-Schiff stain; original magnification, 100x) 


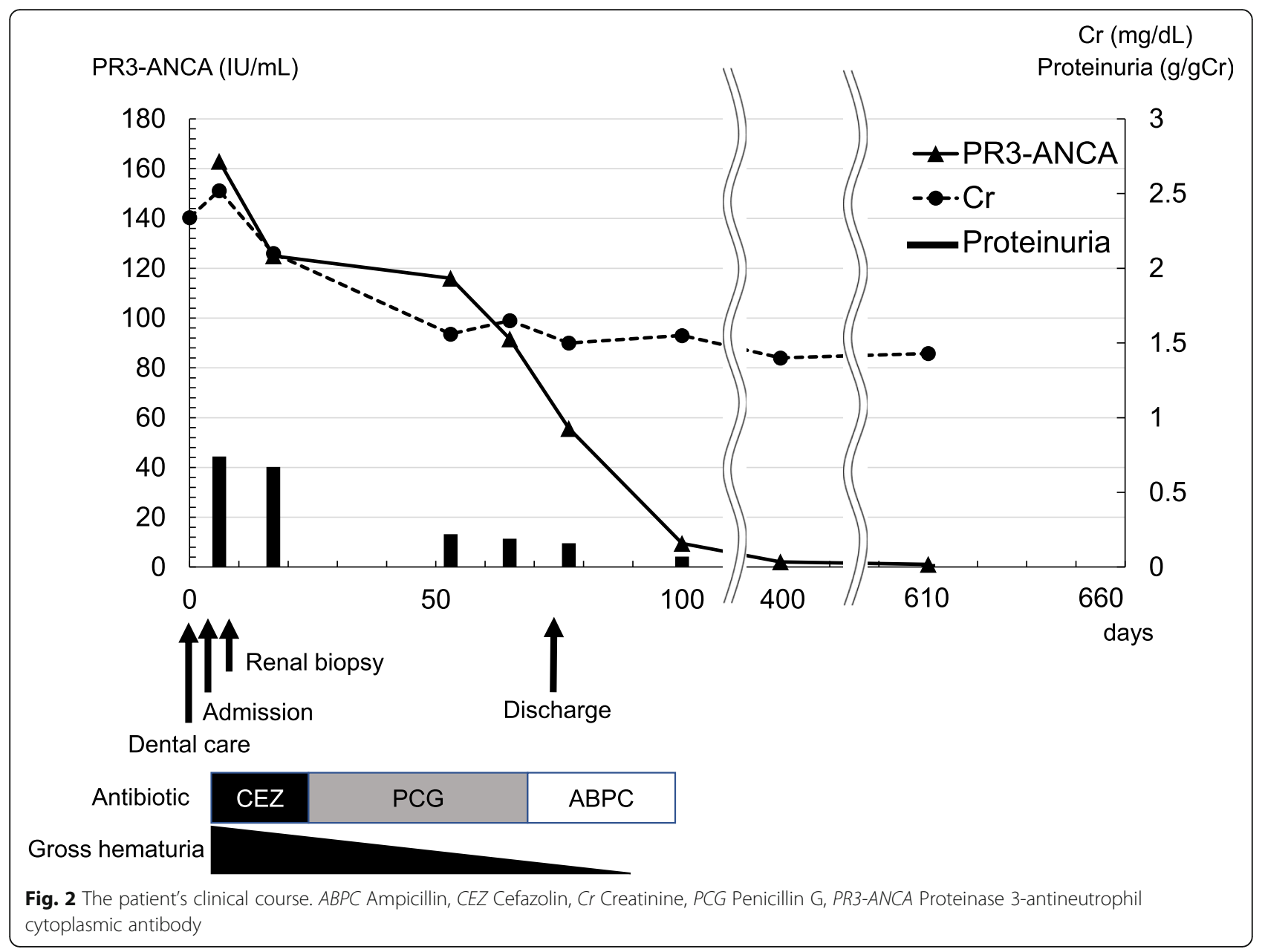

and endothelial cell thickening in the glomerulus and tubulointerstitial damage with infiltration of immune cells [3-7]. PR3-ANCA has been reported to be positive in 5$10 \%$ of cases of renal disease complicated with infective endocarditis [1]. It is considered that PR3-ANCA may be produced as a result of an immune response against infection by sharing epitopes with cytoplasmic antigens of neutrophils in cases of infective endocarditis [8]. The produced PR3-ANCA is then speculated to contribute to fibrinoid necrosis, crescent formation, and granulomas in the kidney [9]. However, the lack of sufficient histological findings of PR3-ANCA-positive renal diseases complicated by infective endocarditis prevents clarification of detailed pathological changes in the kidney. Although many cases of PR3-ANCA-positive renal disease complicated by infective endocarditis have been reported, including crescentic glomerulonephritis, endocapillary proliferative glomerulonephritis, mesangial proliferative glomerulonephritis, and focal segmental glomerulonephritis, only three cases showed necrotizing crescentic glomerulonephritis complicated by infective endocarditis [10-34] (Table 2). Regarding treatment for PR3-ANCA-positive renal disease complicated by infective endocarditis, previous studies suggested antibiotic monotherapy for patients with low PR3-ANCA titers $(<25 \mathrm{IU} / \mathrm{ml})$ and combination therapy with immunosuppressive agents, including steroids for patients with high PR3-ANCA titers (>50 IU/ $\mathrm{ml}$ ), when the patients' condition does not improve with antibiotic monotherapy [22, 35]. The three previous cases of PR3-ANCA-positive necrotizing crescentic glomerulonephritis showed various PR3-ANCA titers $(2.96,>8.0$, and $85 \mathrm{IU} / \mathrm{ml}$ ) and were treated with immunosuppressive agents such as corticosteroids in addition to antibiotics. Among those three cases, the renal disease resolved completely in two patients but progressed to end-stage renal disease in the other (Table 2). The other types of PR3ANCA-positive renal disease complicated by infective endocarditis also showed various PR3-ANCA titers (3$359 \mathrm{IU} / \mathrm{ml}$ ) and were treated with antibiotics with or without immunosuppressive agents (Table 2). Regarding treatment outcomes, most renal diseases recovered, except for one patient with crescentic glomerulonephritis with high PR3-ANCA titers $(247 \mathrm{IU} / \mathrm{ml})$ and one patient with mesangial proliferative glomerulonephritis with high PR3- 
Table 2 Case reports of PR3-ANCA-positive renal injury complicated by infective endocarditis

\begin{tabular}{|c|c|c|c|c|c|c|c|}
\hline $\begin{array}{l}\text { Age } \\
\text { (years)/ } \\
\text { sex }\end{array}$ & $\begin{array}{l}\text { Renal biopsy histology (IF/ } \\
\text { EM) }\end{array}$ & $\begin{array}{l}\text { PR3- } \\
\text { ANCA } \\
(I U / m L)\end{array}$ & $\begin{array}{l}\text { Microbe } \\
\text { detected }\end{array}$ & $\begin{array}{l}\text { Past medical } \\
\text { history }\end{array}$ & Treatments & Outcome & Reference \\
\hline $54 / \mathrm{M}$ & $\begin{array}{l}\text { Focal necrotizing } \\
\text { crescentic GN } \\
\text { (negative/no deposits) }\end{array}$ & 2.96 & $\begin{array}{l}\text { Streptococcus } \\
\text { mutans }\end{array}$ & MVP & $\begin{array}{l}\text { Piperacillin, tazobactam, } \\
\text { cyclophosphamide, } \\
\text { corticosteroids }\end{array}$ & $\begin{array}{l}\text { Complete } \\
\text { recovery }\end{array}$ & [10] \\
\hline $59 / \mathrm{M}$ & $\begin{array}{l}\text { Focal necrotizing } \\
\text { crescentic GN } \\
\text { (negative/no deposits) }\end{array}$ & $>8.0$ & $\begin{array}{l}\text { Enterococcus } \\
\text { faecalis }\end{array}$ & Hepatitis B and C & $\begin{array}{l}\text { Pulse methylprednisolone } \Rightarrow \\
\text { prednisolone }\end{array}$ & Hemodialysis & [11] \\
\hline $67 / M$ & $\begin{array}{l}\text { Focal necrotizing } \\
\text { crescentic } \mathrm{GN} \\
\left(\mathrm{IgA}^{+}, \mathrm{IgM}^{+}, \mathrm{IgG}^{+}, \mathrm{C}^{+},\right. \\
\mathrm{Clq}^{+} / \mathrm{mesangial} \text { and } \\
\text { subendothelial dense } \\
\text { deposits) }\end{array}$ & 85 & $\begin{array}{l}\text { Gemella } \\
\text { sanguinis }\end{array}$ & No mention & $\begin{array}{l}\text { Ceftriaxone, gentamicin, } \\
\text { methylprednisolone }\end{array}$ & $\begin{array}{l}\text { Temporary } \\
\text { hemodialysis } \Rightarrow \\
\text { complete } \\
\text { recovery }\end{array}$ & [12] \\
\hline $6 / M$ & $\begin{array}{l}\text { Crescentic GN } \\
\text { (no mention) }\end{array}$ & Positive & $\begin{array}{l}\text { Bartonella } \\
\text { henselae }\end{array}$ & $\mathrm{CHD}$ & Doxycycline, rifampicin & No mention & [13] \\
\hline $12 / F$ & $\begin{array}{l}\text { Crescentic GN } \\
\text { (C3 } 3^{+} / \text {subendothelial dense } \\
\text { deposits) }\end{array}$ & Positive & $\begin{array}{l}\text { Gemella } \\
\text { morbillorum }\end{array}$ & Nothing & Penicillin, gentamicin, steroids & $\begin{array}{l}\text { Complete } \\
\text { recovery }\end{array}$ & [14] \\
\hline $14 / \mathrm{M}$ & $\begin{array}{l}\text { Crescentic GN } \\
\text { (no mention) }\end{array}$ & Positive & $\begin{array}{l}\text { Bartonella } \\
\text { henselae }\end{array}$ & $\mathrm{CHD}$ & Doxycycline & No mention & [13] \\
\hline $18 / F$ & $\begin{array}{l}\text { Crescentic GN } \\
\text { (lgG }[1+], \text { IgM[3+], C3[2+], } \\
\text { C1q[2+]/subendothelial } \\
\text { dense deposits) }\end{array}$ & 32 & $\begin{array}{l}\text { Bartonella } \\
\text { henselae }\end{array}$ & $\begin{array}{l}\text { Tetralogy of } \\
\text { Fallot, SSS, and } \\
\text { complete heart } \\
\text { block }\end{array}$ & $\begin{array}{l}\text { Antibiotic therapy, } \\
\text { methylprednisolone }\end{array}$ & $\begin{array}{l}\text { Complete } \\
\text { recovery }\end{array}$ & [15] \\
\hline $24 / \mathrm{M}$ & $\begin{array}{l}\text { Crescentic GN } \\
\left(\mathrm{C} 3^{+} / \text {mesangial and }\right. \\
\text { subendothelial dense } \\
\text { deposits) }\end{array}$ & 14 & $\begin{array}{l}\text { a-Hemolytic } \\
\text { Streptococcus }\end{array}$ & VSD & Cefotaxime, prednisolone & $\begin{array}{l}\text { No renal } \\
\text { dysfunction }\end{array}$ & [16] \\
\hline $26 / F$ & $\begin{array}{l}\text { Crescentic GN } \\
\left(\operatorname{lgM}^{+} \mathrm{C} 3^{+} / \text {no mention }\right)\end{array}$ & 160 & $\begin{array}{l}\text { Streptococcus } \\
\text { viridans }\end{array}$ & $\begin{array}{l}\text { ASD, recent } \\
\text { dental Tx }\end{array}$ & Penicillin G, tobramycin & Recovery & [17] \\
\hline $36 / M$ & $\begin{array}{l}\text { Crescentic GN } \\
\text { (negative/no mention) }\end{array}$ & 359 & $\begin{array}{l}\text { Bartonella } \\
\text { henselae }\end{array}$ & No mention & $\begin{array}{l}\text { Prednisolone, intravenous } \\
\text { cyclophosphamide } \Rightarrow \\
\text { azathioprine, MMF, prednisolone, } \\
\text { CV surgery }\end{array}$ & $\begin{array}{l}\text { Complete } \\
\text { recovery }\end{array}$ & [18] \\
\hline $43 / \mathrm{M}$ & $\begin{array}{l}\text { Crescentic GN } \\
\text { (negative/no mention) }\end{array}$ & Positive & $\begin{array}{l}\text { Bartonella } \\
\text { henselae }\end{array}$ & $\begin{array}{l}\text { Infective } \\
\text { endocarditis } \\
\text { (blood culture } \\
\text { was negative) }\end{array}$ & $\begin{array}{l}\text { Cyclophosphamide, } \\
\text { prednisoloneCV surgery }\end{array}$ & No mention & [19] \\
\hline $46 / M$ & $\begin{array}{l}\text { Crescentic GN } \\
\left(\mathrm{C}^{+} \mathrm{C} 1 \mathrm{q}^{+} / \text {no mention }\right)\end{array}$ & 25 & Negative & No mention & Amoxicillin, gentamicin, penicillin & $\begin{array}{l}\text { Complete } \\
\text { recovery }\end{array}$ & [20] \\
\hline $47 / M$ & $\begin{array}{l}\text { Crescentic GN } \\
\left(\operatorname{lgM}^{+}, \operatorname{lgA} A^{+}, \mathrm{C}^{+}, \mathrm{C} \mathrm{q}^{+} /\right. \\
\text {mesangial and } \\
\text { subendothelial dense } \\
\text { deposits) }\end{array}$ & 160 & $\begin{array}{l}\text { Bartonella } \\
\text { henselae }\end{array}$ & $\begin{array}{l}\text { Cat scratch } \\
\text { disease }\end{array}$ & Doxycycline, rifampicin 6 weeks & Recovery & [21] \\
\hline $50 / \mathrm{M}$ & $\begin{array}{l}\text { Crescentic GN } \\
\text { (negative/not performed) }\end{array}$ & 247 & $\begin{array}{l}\text { Streptococcus } \\
\text { oralis }\end{array}$ & Nothing & $\begin{array}{l}\text { Steroid therapy } \Rightarrow \text { ampicillin, } \\
\text { gentamicin, vancomycin }\end{array}$ & Death & [22] \\
\hline $54 / \mathrm{M}$ & $\begin{array}{l}\text { Crescentic GN } \\
\left(\operatorname{lgM}^{+}, C 3^{+} / \text {no deposits) }\right.\end{array}$ & 3 & $\begin{array}{l}\text { Streptococcus } \\
\text { mutans }\end{array}$ & No mention & $\begin{array}{l}\text { Ampicillin } \Rightarrow \text { vancomycin, } \\
\text { corticosteroids, } \\
\text { cyclophosphamide }\end{array}$ & Recovery & [23] \\
\hline $55 / \mathrm{M}$ & $\begin{array}{l}\text { Crescentic GN } \\
\left(C 3[2+], \lg A^{+} / \text {no deposits }\right)\end{array}$ & $>8.0$ & $\begin{array}{l}\text { Bartonella } \\
\text { henselae, } \\
\text { Bartonella } \\
\text { quintana }\end{array}$ & Depression & $\begin{array}{l}\text { Vancomycin, cefepime } \Rightarrow \\
\text { doxycycline, rifampicin, } \\
\text { methylprednisolone }\end{array}$ & Recovery & [24] \\
\hline $67 / M$ & $\begin{array}{l}\text { Crescentic GN } \\
\left(\mathrm{lgM}^{+}, \mathrm{C}^{+}, \mathrm{Clq}^{+} / \mathrm{no}\right. \\
\text { mention) }\end{array}$ & 41 & $\begin{array}{l}\text { Bartonella } \\
\text { henselae }\end{array}$ & $\begin{array}{l}\text { Thoracic aortic } \\
\text { aneurysm repair }\end{array}$ & $\begin{array}{l}\text { Rifampicin, doxycycline, } \\
\text { methylprednisolone }\end{array}$ & $\begin{array}{l}\text { Temporary } \\
\text { hemodialysis } \Rightarrow \\
\text { recovery }\end{array}$ & [25] \\
\hline
\end{tabular}


Table 2 Case reports of PR3-ANCA-positive renal injury complicated by infective endocarditis (Continued)

\begin{tabular}{|c|c|c|c|c|c|c|c|}
\hline $\begin{array}{l}\text { Age } \\
\text { (years)/ } \\
\text { sex }\end{array}$ & $\begin{array}{l}\text { Renal biopsy histology (IF/ } \\
\text { EM) }\end{array}$ & $\begin{array}{l}\text { PR3- } \\
\text { ANCA } \\
(I U / m L) \\
\end{array}$ & $\begin{array}{l}\text { Microbe } \\
\text { detected }\end{array}$ & $\begin{array}{l}\text { Past medical } \\
\text { history }\end{array}$ & Treatments & Outcome & Reference \\
\hline $72 / F$ & $\begin{array}{l}\text { Crescentic GN } \\
\text { (no mention) }\end{array}$ & Positive & $\begin{array}{l}\text { Aggregatibacter } \\
\text { aphrophilus }\end{array}$ & No mention & Vancomycin, ceftriaxone & $\begin{array}{l}\text { Temporary } \\
\text { hemodialysis } \Rightarrow \\
\text { recovery }\end{array}$ & [26] \\
\hline $42 / M$ & $\begin{array}{l}\text { Diffuse endocapillary } \\
\text { proliferative GN } \\
\text { (C } 3^{+} / \text {subendothelial dense } \\
\text { deposits) }\end{array}$ & 21.3 & $\begin{array}{l}\text { Staphylococcus } \\
\text { aureus }\end{array}$ & Nothing & Cefazolin & Recovery & {$[27]$} \\
\hline 68/M & $\begin{array}{l}\text { Diffuse endocapillary } \\
\text { proliferative GN and } \\
\text { crescentic GN } \\
\text { (lgG }[2+], \text { IgM[3+], C3[3+], } \\
\text { C1q[2+]/subendothelial } \\
\text { dense deposits) }\end{array}$ & 102 & Negative & Schistosomiasis & Cefoperazone, tazobactam & $\begin{array}{l}\text { Complete } \\
\text { recovery }\end{array}$ & {$[28]$} \\
\hline $78 / F$ & $\begin{array}{l}\text { Endocapillary proliferative } \\
\text { GN } \\
\left(\mathrm{lgM}^{+}, \mathrm{C}^{+}, \mathrm{C}^{+} \mathrm{q}^{+} / \mathrm{no}\right. \\
\text { mention) }\end{array}$ & 30 & $\begin{array}{l}\text { Bartonella } \\
\text { henselae }\end{array}$ & Hypertension & Doxycycline & $\begin{array}{l}\text { Complete } \\
\text { recovery }\end{array}$ & [29] \\
\hline $48 / \mathrm{M}$ & $\begin{array}{l}\text { Mesangial proliferative GN } \\
\left(\mathrm{C}^{+} / \text {no mention) }\right.\end{array}$ & 12 & Negative & Alcoholism, DM & Amoxicillin, gentamicin & $\begin{array}{l}\text { Complete } \\
\text { recovery }\end{array}$ & {$[20]$} \\
\hline $57 / \mathrm{M}$ & $\begin{array}{l}\text { Mesangial proliferative GN } \\
\left(\mathrm{lgG}^{+}, \operatorname{lgM} M^{+}, \mathrm{C}^{+} / \mathrm{no}\right. \\
\text { mention) }\end{array}$ & 45 & Negative & Nothing & $\begin{array}{l}\text { Corticosteroids } \Rightarrow \text { ampicillin, } \\
\text { ceftriaxone, gentamicin, } \\
\text { vancomycin }\end{array}$ & Recovery & {$[30]$} \\
\hline 74/M & $\begin{array}{l}\text { Mesangial proliferative GN } \\
\left(\mathrm{lgG}^{+}, \mathrm{Clq}^{+} / \text {not }\right. \\
\text { performed) }\end{array}$ & $>100$ & $\begin{array}{l}\text { Bartonella } \\
\text { henselae, } \\
\text { Bartonella } \\
\text { quintana }\end{array}$ & $\begin{array}{l}\text { IHD, pacemaker, } \\
\text { DM, pulmonary } \\
\text { embolus }\end{array}$ & Antibiotic therapy & Recovery & {$[31]$} \\
\hline 78/M & $\begin{array}{l}\text { Mesangial proliferative GN } \\
\left(\mathrm{IgM}^{+}, \mathrm{C}^{+}, \operatorname{lgA}^{+}, \mathrm{Clq}^{+} /\right. \\
\text {subendothelial dense } \\
\text { deposits) }\end{array}$ & 143 & $\begin{array}{l}\text { Enterococcus } \\
\text { faecalis }\end{array}$ & $\begin{array}{l}\text { Coronary artery } \\
\text { bypass surgery }\end{array}$ & Antibiotic therapy & Death & {$[32]$} \\
\hline $57 / \mathrm{M}$ & $\begin{array}{l}\mathrm{FSGS} \\
\text { (lgM }{ }^{+}, \mathrm{C}^{+} / \text {paramesangial } \\
\text { dense deposits) }\end{array}$ & 40 & Negative & $\begin{array}{l}\mathrm{DM}, \mathrm{AVR} \text {, aortic } \\
\text { aneurysm }\end{array}$ & $\begin{array}{l}\text { Pulse methylprednisolone } \Rightarrow \\
\text { vancomycin, gentamicin, } \\
\text { rifampicin }\end{array}$ & $\begin{array}{l}\text { Plasmapheresis } \Rightarrow \\
\text { recovery }\end{array}$ & [33] \\
\hline 64/M & $\begin{array}{l}\text { FSGS and mild interstitial } \\
\text { inflammation } \\
\text { (no mention) }\end{array}$ & 60 & Negative & $\begin{array}{l}\text { Insidious mild } \\
\text { renal dysfunction }\end{array}$ & Ceftriaxone, doxycycline & $\begin{array}{l}\text { Complete } \\
\text { recovery }\end{array}$ & [34] \\
\hline
\end{tabular}

Abbreviations: $A S D$ atrial septal defect, $A V R$ aortic valve replacement, $C H D$ chronic heart disease, $C 3$ complement component 3, CV cardiovascular, DM diabetes mellitus, EM electron microscopy, F female, FSGS focal segmental glomerulonephritis, GN glomerular nephritis, IF immunofluorescence, IHD ischemic heart disease, Ig immunoglobulin, $L M$ light microscopy, $M$ male, MMF mycophenolate mofetil, MVP mitral valve prolapse, PR3-ANCA proteinase 3 antineutrophil cytoplasmic antibody, SSS sick sinus syndrome, Tx treatment, VSD ventricular septal defect

ANCA titers $(143 \mathrm{IU} / \mathrm{ml})$, both of whom died (Table 2). In our patient, necrotizing crescentic glomerulonephritis improved with antibiotic monotherapy, and PR3ANCA titer normalized in accordance with improving infective endocarditis; however, PR3-ANCA titer was highly elevated at $163 \mathrm{IU} / \mathrm{ml}$. The results of our patient's case suggest that antibiotic monotherapy can be effective even if the PR3-ANCA titer is considerably high in PR3-ANCA-positive necrotizing crescentic glomerulonephritis complicated by infective endocarditis. However, caution is needed with the use of immunosuppressive agents because they may exacerbate bacteremia and infective endocarditis. Furthermore, a greater accumulation of cases with histological evidence is needed to investigate optimal treatments for PR3-
ANCA-positive renal disease complicated with infective endocarditis.

In conclusion, we describe a case of a patient with PR3-ANCA-positive necrotizing crescentic glomerulonephritis complicated by infective endocarditis. His renal disease was improved with antibiotic agents, and his PR3-ANCA titer normalized in accordance with improving infective endocarditis.

\section{Abbreviations}

ABPC: Ampicillin; ALT: Alanine aminotransferase; ASD: Atrial septal defect; ASO: Antistreptolysin O; AST: Aspartate aminotransferase; AVR: Aortic valve replacement; $\beta_{2}-$ MG: $\beta_{2}-$ Microglobulin; BUN: Blood urea nitrogen; C3: Complement component 3; C4: Complement component 4; CEZ: Cefazolin; CH50: 50\% Homolytic unit of complement; CHD: Chronic heart disease; Cr: Creatinine; CRP: C-reactive protein; CV: Cardiovascular; DM: Diabetes mellitus; eGFR: Estimated glomerular filtration rate; EM: Electron 
microscopy; ESR: Erythrocyte sedimentation rate; F: Female; FSGS: Focal segmental glomerulonephritis; GBM: Antiglomerular basement membrane antibody; GN: Glomerular nephritis; HbA1c: Hemoglobin A1c; HPF: Highpower field; IF: Immunofluorescence; Ig: Immunoglobulin; IHD: Ischemic heart disease; LM: Light microscopy; M: Male; MMF: Mycophenolate mofetil; MPO-ANCA: Myeloperoxidase antineutrophil cytoplasmic antibody; MVP: Mitral valve prolapse; PCG: Penicillin G; PR3-ANCA: Proteinase 3antineutrophil cytoplasmic antibody; RBC: Red blood cells; RNP: Ribonucleoprotein; Sm: Smith; SSS: Sick sinus syndrome; Tx: Treatment; VSD: Ventricular septal defect; WBC: White blood cells

\section{Acknowledgements}

We thank Christina Croney, Ph.D., of Edanz Group (www.edanzediting.com/ ac) for editing a draft of the manuscript.

\section{Authors' contributions}

$\mathrm{KY}$ and $\mathrm{YK}$ wrote the manuscript. $\mathrm{KH}$ and $\mathrm{YM}$ supervised the study. $\mathrm{YU}, \mathrm{MH}$, and YM undertook histological analysis. All authors participated in patient care. All authors read and approved the final manuscript.

\section{Ethics approval and consent to participate}

Approval of the institutional ethics committee was not required, because this is a case report without any experimental trial.

\section{Consent for publication}

Written informed consent was obtained from the patient for publication of this case report and any accompanying images. A copy of the written consent is available for review by the Editor-in-Chief of this journal.

\section{Competing interests}

The authors declare that they have no competing interests.

\section{Author details}

'Division of Nephrology, First Department of Integrated Medicine, Saitama Medical Center, Jichi Medical University, 1-847 Amanuma-cho, Omiya-ku, Saitama, Saitama 330-8503, Japan. ²Division of Pathology, First Department of Integrated Medicine, Saitama Medical Center, Jichi Medical University, Saitama, Japan. ${ }^{3}$ Department of Diagnostic Pathology, Dokkyo University Koshigaya Medical Center, Saitama, Japan.

Received: 13 June 2019 Accepted: 2 October 2019

Published online: 05 December 2019

\section{References}

1. Boils CL, Nasr SH, Walker PD, Couser WG, Larsen CP. Update on endocarditis-associated glomerulonephritis. Kidney Int. 2015;87:1241-9.

2. Pierce D, Calkins BC, Thornton K. Infectious endocarditis: diagnosis and treatment. Am Fam Physician. 2012;85:981-6.

3. Nasr SH, Radhakrishnan J, D'Agati VD. Bacterial infection-related glomerulonephritis in adults. Kidney Int. 2013;83:792-803.

4. Kannan S, Mattoo TK. Diffuse crescentic glomerulonephritis in bacterial endocarditis. Pediatr Nephrol. 2001;16:423-8.

5. Toth T. Crescentic involved glomerulonephritis in infective endocarditis. Int Urol Nephrol. 1990;22:77-88.

6. Orfila C, Lepert JC, Modesto A, Goudable C, Suc JM. Rapidly progressive glomerulonephritis associated with bacterial endocarditis: efficacy of antibiotic therapy alone. Am J Nephrol. 1993;13:218-22.

7. Neugarten J, Gallo GR, Baldwin DS. Glomerulonephritis in bacteria endocarditis. Am J Kidney Dis. 1984;3:371-9.

8. Collazos J, Diaz F, Mayo J, Martinez E. Infectious endocarditis, vasculitis, and glomerulonephritis. Clin Infect Dis. 1999;28:1342-3.

9. Hilhorst M, Shirai T, Berry G, Goronzy JJ, Weyand CM. T cell-macrophage interactions and granuloma formation in vasculitis. Front Immunol. 2014;5:432.

10. Konstantinov KN, Harris AA, Hartshorne MF, Tzamaloukas AH. Symptomatic anti-neutrophil cytoplasmic antibody-positive disease complicating subacute bacterial endocarditis: to treat or not to treat? Case Rep Nephrol Urol. 2012;2:25-32.

11. Cervi A, Kelly D, Alexopoulou I, Khalidi N. ANCA-associated pauci-immune glomerulonephritis in a patient with bacterial endocarditis: a challenging clinical dilemma. Clin Nephrol Case Stud. 2017;5:32-7.
12. Rousseau-Gagnon M, Riopel J, Desjardins A, Garceau D, Agharazii M, Desmeules S. Gemella sanguinis endocarditis with c-ANCA/anti-PR-3associated immune complex necrotizing glomerulonephritis with a 'fullhouse' pattern on immunofluorescence microscopy. Clin Kidney J. 2013;6: 300-4.

13. Ouellette $\mathrm{CP}$, Joshi $\mathrm{S}$, Texter $\mathrm{K}$, Jaggi P. Multiorgan involvement confounding the diagnosis of Bartonella henselae infective endocarditis in children with congenital heart disease. Pediatr Infect Dis J. 2017;36:516-20.

14. Kumar G, Al Ali AS, Gulzar Bhatti N. Rare bacteria infecting the heart and affecting the kidney of a young child. Case Rep Nephrol Dial. 2017;7:138-43.

15. Khalighi MA, Nguyen S, Wiedeman JA, Palma Diaz MF. Bartonella endocarditis-associated glomerulonephritis: a case report and review of the literature. Am J Kidney Dis. 2014;63:1060-5.

16. Fukuda M, Motokawa M, Usami T, Oikawa T, Morozumi K, Yoshida A, Kimura G. PR3-ANCA-positive crescentic necrotizing glomerulonephritis accompanied by isolated pulmonic valve infective endocarditis, with reference to previous reports of renal pathology. Clin Nephrol. 2006;66:202-9.

17. Wagner J, Andrassy K, Ritz E. Is vasculitis in subacute bacterial endocarditis associated with ANCA? Lancet. 1991;337:799-800.

18. Shah SH, Grahame-Clarke C, Ross CN. Touch not the cat bot a glove: ANCApositive pauci-immune necrotizing glomerulonephritis secondary to Bartonella henselae. Clin Kidney J. 2014;7:179-81.

19. Vikram HR, Bacani AK, DeValeria PA, Cunningham SA, Cockerill FR 3rd. Bivalvular Bartonella henselae prosthetic valve endocarditis. J Clin Microbiol. 2007:45:4081-4

20. Subra JF, Michelet C, Laporte J, Carrere F, Reboul P, Cartier F, Saint-Andre JP, Chevailler A. The presence of cytoplasmic antineutrophil cytoplasmic antibodies (C-ANCA) in the course of subacute bacterial endocarditis with glomerular involvement, coincidence or association? Clin Nephrol. 1998;49:15-8.

21. Vercellone J, Cohen L, Mansuri S, Zhang PL, Kellerman PS. Bartonella endocarditis mimicking crescentic glomerulonephritis with PR3-ANCA positivity. Case Rep Nephrol. 2018;2018:9607582.

22. Kishimoto N, Mori Y, Yamahara H, Kijima Y, Nose A, Uchiyama-Tanaka Y, Tokoro T, Nagata T, Umeda Y, Takahashi N, et al. Cytoplasmic antineutrophil cytoplasmic antibody positive pauci-immune glomerulonephritis associated with infectious endocarditis. Clin Nephrol. 2006;66:447-54.

23. Zeledon Jl, McKelvey RL, Servilla KS, Hofinger D, Konstantinov KN, Kellie S, Sun Y, Massie LW, Hartshorne MF, Tzamaloukas AH. Glomerulonephritis causing acute renal failure during the course of bacterial infections: histological varieties, potential pathogenetic pathways and treatment. Int Urol Nephrol. 2008;40:461-70.

24. Raybould JE, Raybould AL, Morales MK, Zaheer M, Lipkowitz MS, Timpone JG, Kumar PN. Bartonella endocarditis and pauci-immune glomerulonephritis: a case report and review of the literature. Infect Dis Clin Pract (Baltim Md). 2016;24:254-60.

25. Van Haare HS, Wilmes D, Aydin S, Clerckx C, Labriola L. Necrotizing ANCApositive glomerulonephritis secondary to culture-negative endocarditis. Case Rep Nephrol. 2015;2015:649763.

26. Hirano K, Tokui T, Inagaki M, Fujii T, Maze Y, Toyoshima H. Aggregatibacter aphrophilus infective endocarditis confirmed by broad-range PCR diagnosis: a case report. Int J Surg Case Rep. 2017;31:150-3.

27. Kawamorita $Y$, Fujigaki $Y$, Imase A, Arai S, Tamura $Y$, Tanemoto M, Uozaki $H$, Yamaguchi $Y$, Uchida S. Successful treatment of infectious endocarditis associated glomerulonephritis mimicking C3 glomerulonephritis in a case with no previous cardiac disease. Case Rep Nephrol. 2014;2014:569047.

28. Peng H, Chen WF, Wu C, Chen YR, Peng B, Paudel SD, Lou TQ. Culturenegative subacute bacterial endocarditis masquerades as granulomatosis with polyangiitis (Wegener's granulomatosis) involving both the kidney and lung. BMC Nephrol. 2012:13:174.

29. Salvado C, Mekinian A, Rouvier P, Poignard P, Pham I, Fain O. Rapidly progressive crescentic glomerulonephritis and aneurism with antineutrophil cytoplasmic antibody: Bartonella henselae endocarditis. Presse Med. 2013;42: 1060-1.

30. Reza Ardalan M, Trillini M. Infective endocarditis mimics ANCA associated glomerulonephritis. Caspian J Intern Med. 2012;3:496-9.

31. Forbes SH, Robert SC, Martin JE, Rajakariar R. Quiz page January 2012: Acute kidney injury with hematuria, a positive ANCA test, and low levels of complement. Am J Kidney Dis. 2012;59:A28-31.

32. MCAdoo SP, Densem C, Salama A, Pusey CD. Bacterial endocarditis associated with proteinase 3 anti-neutrophil cytoplasm antibody. NDT Plus. 2011:4:208-10. 
33. Mohandes S, Satoskar A, Hebert L, Ayoub I. Bacterial endocarditis manifesting as autoimmune pulmonary renal syndrome: ANCA-associated lung hemorrhage and pauci-immune crescentic glomerulonephritis. Clin Nephrol. 2018;90:431-3.

34. Sugiyama H, Sahara M, Imai Y, Ono M, Okamoto K, Kikuchi K, Nagai R. Infective endocarditis by Bartonella quintana masquerading as antineutrophil cytoplasmic antibody-associated small vessel vasculitis. Cardiology. 2009;114:208-11.

35. Haseyama T, Imai H, Komatsuda A, Hamai K, Ohtani H, Kibira S, Miura AB. Proteinase-3-antineutrophil cytoplasmic antibody (PR3-ANCA) positive crescentic glomerulonephritis in a patient with Down's syndrome and infectious endocarditis. Nephrol Dial Transplant. 1998;13:2142-6.

\section{Publisher's Note}

Springer Nature remains neutral with regard to jurisdictional claims in published maps and institutional affiliations.

Ready to submit your research? Choose BMC and benefit from:

- fast, convenient online submission

- thorough peer review by experienced researchers in your field

- rapid publication on acceptance

- support for research data, including large and complex data types

- gold Open Access which fosters wider collaboration and increased citations

- maximum visibility for your research: over $100 \mathrm{M}$ website views per year

At BMC, research is always in progress.

Learn more biomedcentral.com/submissions 